\title{
ACERCA DE CIERTA EXPERIENCIA DE LO HISTÓRICO EN HEGEL
}

\author{
Francisco Abalo Cea ${ }^{1}$
}

RESUMEN: El siguiente artículo intenta reflexionar acerca de los límites de la Filosofía de la Historia en el pensamiento de Hegel. Con este propósito, el esfuerzo se concentra en la interpretación de una cierta experiencia de lo histórico que nos presenta Hegel en su "Introducción a la Filosofía de la Historia”. La exposición que hace el autor exhibe ciertos elementos que están vinculados al tratamiento filosófico del asunto. Por otra parte, se muestra que la misma justificación de la Filosofía de la Historia aparece bosquejada en este pasaje. Por último se introduce la discusión acerca de algunos aspectos problemáticos en el planteamiento hegeliano tomando en cuenta los resultados de esta interpretación.

PALABRAS CLAVE: Filosofía de la Historia. Historia. Experiencia. Espíritu. Hegel.

\section{INTRODUCCIÓN}

El presente trabajo se centrará en una peculiar experiencia de lo histórico que nos presenta Hegel en su "Introducción" a la "Filosofía de la Historia"2. El propósito consiste primeramente en mostrar, a través de una interpretación de dicho pasaje, que en la selección y exposición de dicha experiencia se hacen visibles ciertos elementos característicos del planteamiento hegeliano de la Filosofía de la Historia. En segundo lugar, intentaremos desarrollar cómo es que en conexión con dicha experiencia Hegel esboza una cierta justificación de la Filosofía de la Historia en el marco de su pensamiento. Por último, se intentará abrir la discusión acerca de si esta experiencia de lo histórico es lo suficientemente radical y, por consecuencia, el planteamiento filosófico relacionado a ella está radicalmente legitimado, o si no hay más bien aquí una cierta insuficiencia que constituye la posible base para un auténtico trabajo crítico en el modo de abordar el asunto.

\footnotetext{
${ }^{1}$ Universidad de Chile - E-mail: fjabalocea@gmail.com

${ }^{2}$ Editado por Johannes Hoffmeister con el título Die Vernunft in der Geschichte (HEGEL, 1955).
} 
Sin embargo, antes de entrar propiamente en la interpretación, cabe hacer algunas advertencias preliminares para el mejor entendimiento de lo que me he propuesto desarrollar.

En primer lugar, no es el propósito principal del ensayo exponer la concepción hegeliana de la historia, lo cual supondría una investigación exhaustiva de dicho concepto en los distintos contextos en que aparece en el sistema. Con esto también quedan en suspenso los vínculos de fundamentación y de dependencia que en el propio sistema hegeliano permiten una apropiación acabada del concepto de historia. Tampoco está en el centro de la exposición la discusión de la función y relevancia de lo histórico en la determinaciones fundamentales del sistema o de alguna de sus dimensiones decisivas, como por ejemplo en la interpretación que hace Hyppolite de la "Fenomenología del Espíritu” (HYPPOLITE, 1994), Falke (FALKE, 1996) o incluso la de Marcuse en relación a la "Ciencia de la Lógica" (MARCUSE, 1932). Pero, por último, estas advertencias no implican necesariamente que estos planteamientos estén de plano invalidados y que lo que se intenta desarrollar no tenga ninguna relación con estos problemas, sino más bien que para el esclarecimiento del tema que consideraré, no constituyen el propósito esencial y, por tanto, no será el punto de partida. Por eso, la cuestión de si lo que toca tratar aquí se vincula de alguna manera con este conjunto de asuntos puede quedar indeterminada.

Ahora bien, despejada provisionalmente esta cuestión parece legítimo preguntar: ¿de qué se trata entonces? La respuesta está indicada en el título, lo que nos lleva a una última y decisiva advertencia inicial. Se trata de cierta experiencia de lo histórico que podemos encontrar en Hegel. Pero con "experiencia" no se mienta todavía el rigoroso concepto dialéctico de experiencia, es decir, el concepto tal como Hegel lo acuña en el Prólogo de la "Fenomenología del Espíritu" (HEGEL, 2005, p. 80-81). Más bien, apelo a un sentido más laxo, a saber, que el "experimentar" comprende una referencia directa a lo experimentado de tal forma que en ese mismo experimentar es accesible de algún modo esta misma "referencia". Esta caracterización primaria no implica necesariamente que se trate de manera exclusiva de experiencias de tipo "sensible", pero tampoco sólo de "otro tipo". Sin embargo, esta somera caracterización, ciertamente muy formal por el momento, incluye por lo pronto la "experiencia de las cosas" en un plano pre-filosófico y pre-científico, es decir, en la forma más habitual del "experimentar". En este sentido, si se quiere todavía vago, es como debiésemos entender en el presente contexto el 
término "experiencia”. De ahí que hablemos cautamente de cierta experiencia de lo histórico ${ }^{3}$.

\section{EL TESTIMONIO DE CIERTA EXPERIENCIA DE LO HISTÓRICO EN LA “INTRODUCCIÓN" a la "Filosofía de la Historia” de Hegel}

A partir de la advertencia anterior parece natural preguntar: ¿se puede hablar en Hegel de tal "experiencia"? Para responder me remitiré a un pasaje concreto en el esta experiencia de lo histórico salta a la vista como algo anterior a toda conformación filosófica del asunto. Transcribo a continuación este pasaje por extenso en una versión propia:

Si contemplamos el espectáculo de las pasiones humanas y fijamos nuestros ojos en las consecuencias históricas de su violencia, de la insensatez que no sólo acompaña a estas pasiones, sino que incluso y de modo preferente a las buenas intenciones y fines rectos; si contemplamos el mal, la perversidad, la decadencia de los reinos más florecientes que el espíritu humano ha podido producir; si miramos a los individuos con la más profunda compasión por su innombrable miseria, entonces hemos de acabar lamentando con tristeza esta caducidad en general en tal espectáculo y, en tanto este irse a pique no es sólo obra de la naturaleza, sino de la voluntad humana, lamentarlo incluso con tristeza moral, con la molestia del buen espíritu, si tal habita en nosotros. Uno podría realzar en el cuadro más tremendo estas consecuencias sin exageración retórica, sólo juntando correctamente las desgracias que ha sufrido lo más elevado en las creaciones de pueblos y estados como en las virtudes privadas o a lo menos en la inocencia de los individuos, y así mismo llevar el sentimiento hasta la más profunda y desazonada tristeza, que ningún resultado consolador podría contrapesar. Contra esta tristeza siempre podremos fortificarnos o por lo menos huir de ella si pensamos algo como que "así ha sido", "es el destino", "no hay nada que se pueda cambiar", para entonces salir del tedio que esta reflexión de la tristeza puede hacernos y meternos de nuevo en nuestro sentimiento vital, en el presente de nuestras metas e intereses, los cuales exigen no la tristeza por la caducidad, sino nuestra actividad. O tal vez refugiarnos en el egoísmo, que permanece en la orilla tranquila, y contempla seguro desde ahí la lejana visión de la masa confusa de ruinas. Pero incluso en tanto consideremos la historia como el banco de verdugo en el cual han sido llevados a sacrificio la dicha de los pueblos, la sabiduría de los Estados y la virtud de los individuos, así le surge necesariamente al

\footnotetext{
${ }^{3}$ El sentido aquí implicado de "experiencia" es más afín a su uso fenomenológico-hermenéutico que al que se tiene en la orientación empirista de la filosofía, por lo menos en su versión clásica en Locke o Hume. Para mayor ahondamiento de este sentido, consúltese M. HEIDEGGER (1995, p. 9-14).
} 
pensamiento también la pregunta: ¿para quién, para qué meta ha sido ofrecido este sacrificio inmenso? Aquí es donde habitualmente se plantea la pregunta por aquello que ha constituido el inicio general de nuestras consideraciones [...]. ${ }^{4}$ (HEGEL, 1955, p. 79-80, traducido del original).

La interpretación de estos pasajes requiere de poner de relieve algunos aspectos generales. Además, estos nos servirán de guía para alcanzar un nivel de mayor concreción en el presente intento.

En primer lugar, conviene tener en cuenta el contexto al que pertenece el pasaje recién citado. En la edición de Hoffmeister al menos, este pasaje aparece en la sección [B] ("Die Verwirklichung des Geistes") de la introducción (HEGEL, 1955, p. 50-148) y, más precisamente, a lo que en esta edición se ha subdividido bajo el nombre de "Los medios de la realización" ("Die Mittel der Verwilklichung”, HEGEL, 1955, p. 78-110). Esto es importante porque, en cierto sentido, es la exposición de esta experiencia la que nos da cuenta de aquello que dentro del planteamiento filosófico del asunto debe ser considerado como "medio". Por qué ha de ser considerado filosófico-categorialmente así, es algo que no nos dice el pasaje; tampoco bajo qué perspectiva tiene sentido hablar en el tratamiento filosófico de la historia de algo así como "medios". Por otra parte, dentro de la fijación contextual del pasaje, éste tiene un antecedente en un pasaje anterior de la sección [A] ("Ihr allgemeine Begriff" (HEGEL, 1955 , p. 28-29), aun cuando entre ambos hay diferencias relevantes.

En segundo lugar, tenemos que considerar, en una dirección más decisiva aún, qué es aquí lo que toma voz. Se trata de lo que podríamos nombrar sin mucha violencia la "conciencia corriente" . Se trata de la forma de experimentar lo histórico pre-filosófica o, lo que es lo mismo, pre-científica. Nótese con especial atención el "habitualmente" (gewöhnlich) que aparece en la frase final del extracto citado. Lo importante, sin embargo, es que esta experiencia de la conciencia corriente con lo histórico es introducida, entre otras cosas, en relación al "inicio general" de la Filosofía de la Historia; dicho de otra manera, esta experiencia habla aquí con respecto a algo y en la medida en que nos permita vislumbrar algo relevante en relación al verdadero punto

\footnotetext{
${ }^{4}$ Parece bien atestiguado que todo este pasaje esta a lo menos inspirado en las lecturas que hace Hegel de Volney (Voyage en Egypte et Syrie, Paris, 1787). Acerca de la presencia de Volney en el pensamiento hegeliano, consúltese Jacques D’ HONDT (1986, p. 83-113).

${ }^{5}$ Hegel la llama más bien aquí de forma despectiva "trübe Empfindung" que incluye en sí una "sinnende Reflexion" que se lleva a cabo sobre la primera (HEGEL, 1955, p. 80).
} 
de partida de la Filosofía de la Historia, aun cuando esto pueda ser "negativo" para la experiencia expuesta.

Por último, la experiencia consiste en un cierto comportamiento perceptual: se trata de un "contemplar", "mirar". Este marcado carácter "visual" (en sentido lato) de la experiencia condiciona la forma de lo experimentado en una cierta dirección ${ }^{6}$.

Hasta aquí los tres aspectos relevantes que consideraré más concretamente en lo siguiente. Ahora, es necesario avanzar en la interpretación.

\section{INTERPRETACIÓN DE PASAJE}

Tomemos por lo pronto el segundo de los aspectos destacados, pero todavía en son de pregunta. Se puede preguntar entonces: ¿en qué medida la experiencia expuesta está vinculada al "inicio general” de la Filosofía de la Historia? La respuesta a esta pregunta se puede comenzar con el desarrollo de algunos elementos contenidos en el primer aspecto. En otras palabras, es necesario partir por exponer con mayor detalle el contexto en que el anterior pasaje aparece y del cual obtiene su sentido.

El pasaje, como ya se ha dicho, pertenece a la sección [B] de la "Introducción". En esta sección se trata justamente de exponer aquellas condiciones según las cuales cabe entender la realización de lo que para la Filosofía de la Historia se constituye como el "sujeto" del desarrollo histórico universal, a saber, el "Espíritu", y más precisamente, el "Espíritu universal". Justamente la exposición de la historia universal en términos de la "realización del Espíritu universal" es el "objeto" de la investigación filosófica (HEGEL, 1955, p. 29). Una de estas condiciones, por decirlo así, "a priori” del desarrollo de la historia consiste en ser una totalidad que se realiza disponiendo para ello de "medios". De esta forma, la perspectiva que indica Hegel en el tratamiento filosófico de la historia implica algo constituido de tal forma que la realización de la historia y el hecho mismo de que la historia sea "real" (wirklich) refieren a un esquema en donde operan "medios" y "meta" (Endzweck). Lo hago notar por el momento sólo al paso. Si, en cambio, dejamos por ahora de lado la estructura ontológica y gnoseológica que propone Hegel, parece justificado preguntar a qué se refiere el pensador con estos "medios". La respuesta no comporta ninguna

\footnotetext{
${ }^{6}$ Como veremos más adelante en su relevancia, Hegel se refiere insistentemente a la historia universal como un "cuadro" o un "teatro", algo que cabe a lo sumo o por lo menos primeramente contemplar.
} 
dificultad por este lado, ya que precisamente se refiere a los "hechos históricos", incluyendo motivaciones, intereses y consecuencias de estos hechos, es decir, a todo eso que la conciencia corriente accede "sin más" cuando habla de historia. Es por cierto central y muy ilustrativo del planteamiento hegeliano que aquello que se toma "inmediatamente" por histórico sea, sin embargo, considerado a la luz de la categoría de "medio". Pero todavía es necesario avanzar algunos pasos más para abordar este delicado asunto.

Por otra parte, el pasaje citado es muy afín a otro pasaje anterior de la sección [A] ("La historia universal en su concepto"), pero entre ambos hay diferencias considerables. El pasaje de [A] (HEGEL, 1955, p. 34) aparece en el contexto en el que es necesario mostrar ese elemento categorial desde el cual se proyecta la Filosofía de la Historia. Para ello Hegel expone sumariamente las tres categorías que en su articulación dialéctica dan como candidata propicia para ser categoría filosófica a la tercera de ellas (la "meta"). La Filosofía de la Historia considera la historia desde el punto de vista de su "meta", es decir, la historia tiene sentido filosófico cuando es considerada desde el punto de vista de eso hacia donde tiende el conjunto del desarrollo histórico en su despliegue temporo-espiritual. Lo decisivo para nosotros consiste en que nuevamente en este contexto de exposición de "categorías" (HEGEL, 1955, p. 34-36), se muestra cierta experiencia corriente de lo histórico en cuanto ella está fundada primordialmente en la mera categoría de "variación" (Veränderung, HEGEL, 1955, p. 34). Con esto el filósofo quiere asentar principalmente que la "conciencia corriente" y, como consecuencia de ella, la historiografía en curso (historia pragmática) creen atenerse incautamente sólo a "hechos", siendo que ya de entrada en este recalcitrante "empirismo" se introducen elementos conceptuales inmanentes a toda mera observación de los hechos. Ya la multiplicidad que supone ver "hechos" o tan sólo uno de ellos, entendida como sucesión temporal o diversidad en el espacio, es manifiestamente algo anterior a los "hechos" en el seno mismo de la experiencia que se hace de lo histórico. Pero no sólo en este plano se da una prioridad con respecto a los hechos. Este segundo plano es decisivo con respecto a nuestra interpretación, pues Hegel no sólo destaca la ceguera con respecto a estos elementos "abstractos" en toda experiencia de lo histórico, sino que incluso se muestra ingenua en relación al sentido general de lo histórico en cuanto refiere y puede referir a una conciencia (experiencia) considerada en cuanto tal. En otras palabras, toda referencia directa a hechos históricos se da a su vez como cierto "estado de ánimo" que despierta en nosotros en virtud de algo "en” el hecho. Así es como el éxito de determinadas empresas históricas nos producen "alegría”, 
el fracaso en cambio de otras "tristeza" y "dolor". Esto solo es ya el índice de que el "hecho" histórico es de otra constitución que, por ejemplo, un suceso natural; es decir, que en el hecho histórico "algo" nos va de nosotros mismos como para que en su experiencia nos podamos sentir a nosotros mismos de tal o cual manera ${ }^{7}$. Desde este punto de vista, es decisivo para le experiencia de lo histórico y de lo experimentado en ella esa referencia a quien experimenta en tanto en ella él se siente (triste o alegre), es decir, la experiencia de lo histórico está determinada por una referencia hacia si de quien experimenta. Intentaré ahondar en esto último, pero ahora poniendo de relieve la diferencia que aparece en el pasaje citado.

La diferencia reside en que el pasaje de $[B]$ da un paso más allá. Si consideramos ahora el tercero de los aspectos destacados más arriba, se puede comenzar a vislumbrar esta diferencia. En primer lugar, ¿de qué se trata en la experiencia descrita? O mejor, ¿de qué modo es ella experiencia de lo histórico y cómo aparece aquí lo histórico? La experiencia está inmediatamente ante lo histórico en el modo de asistir al espectáculo (Schauspiel) de "las pasiones humanas". En este sentido la experiencia primera que se destaca es aquella que tiene lo histórico ante su mirada, lo "observa" y lo "contempla" como la totalidad de escenas que componen este cuadro en total. Lo importante por esto no es sólo el carácter "visual" con el que parte la experiencia, sino a la vez que lo que ahí se tiene ante la mirada aparece desde ya "universalmente", esto es, como un todo conjunto ${ }^{8}$. La experiencia es ciertamente de algún hecho histórico en particular, pero siempre sobre el fondo del "panorama" de la historia en la cual, como en un espectáculo de teatro o como un cuadro ricamente compuesto, se destaca esto o lo otro del conjunto en que se halla. Por lo tanto, lo importante ahora es que, a diferencia de [A], la experiencia no sólo refiere a un hecho histórico puntual, sino que referida a ello está a su vez remitida a un todo mayor unitario (la historia).

Ahora bien, si lo que está en juego en la experiencia de lo histórico es de alguna manera siempre la "historia universal", entonces ese aspecto "anímico" que Hegel ha destacado antes, se vuelve en nuestro pasaje algo central. Pues, cuando Hegel apunta a esto "que nos pasa" ya no se mueve sólo en la simple relación de esos que observan y eso que es observado en conjunto como un

\footnotetext{
${ }^{7}$ Para mayor ahondamiento, consúltese el pasaje citado: HEGEL (1955, p. 34-35).

${ }^{8}$ Nótese que el término usado por Hegel para hablar de "historia universal" es el de Weltgeschichte, "historia mundial". Empero, por el sentido de las imágenes que expone aquí cabe pensar que "mundo" es algo así como una "totalidad unitaria", es decir, el mundo como totalidad.
} 
espectáculo teatral, sino que más bien pone en primer plano el modo en que nosotros quedamos referidos a nosotros mismos cuando tenemos ante nosotros lo histórico en total. Nosotros quedamos referidos a nosotros mismos en la observación de lo histórico inmediatamente sintiéndonos así o así, en un determinado estado anímico. La introducción de un "estado anímico" no es casual o una nota dramática mediante la cual el autor quisiera darle mayor colorido a su texto. Al contrario, esta introducción es un paso necesario en la dirección que conduce a la apertura del sentido filosófico de la cuestión, que no tiene nada que ver con una interesante constatación psicológica.

Si preguntamos ahora directamente por el estado de ánimo que se abre en la observación de lo histórico, Hegel responde: ahí nos sentimos tristes (traurig). Pero ¿qué significa esto?

Si la descripción que aquí tiene lugar la comparamos con las menciones que se hacen de ésta misma en el contexto del examen de las categorías de lo histórico, encontramos la diferencia que resulta decisiva. Si en ese contexto Hegel puso de relieve que el "espectáculo de la historia" movía, por razones diversas, tanto a la alegría y el entusiasmo como a la tristeza, aquí en cambio se pone en primera línea sólo este último estado anímico. Pues, a diferencia de esta alegría, que se regocija con el éxito de tal empresa o con la realización de tal propósito, en esta tristeza no sólo nos apenamos por el fracaso de tal intento o la desaparición de tal pueblo, sino que "hemos de acabar lamentando con tristeza esta caducidad en general". Lo relevante en esta "tristeza" consiste en que se pone al descubierto el carácter "pretérito", o mejor, caduco (Vergängligkeit) de todo acontecimiento histórico como tal, es decir, que todo intento, empresa o conquista está en la historia, independiente de su éxito o fracaso real, destinado finalmente a desaparecer, a caer en la insignificancia y en el completo olvido. Nada en verdad permanece ni queda, cuando tiene lugar la marcha (Gang) de la historia, sino que en su mismo nacimiento todo lo histórico porta en sí el destino de ser-pasado (ver-gangene), de irse a pique (unter-gehen) por muy nobles o virtuosos que sean sus propósitos perseguidos o incluso alcanzados. Nos entristecemos porque frente a este poderoso movimiento negador de la historia, todo hecho histórico como tal consiste sólo en pasar (gehen) y lo histórico en conjunto sólo muestra esta desazonadora verdad: frente a la negación que es en sí misma la historia ningún acontecimiento histórico puede quedar en pie. Lo que la experiencia de lo histórico descubre en la tristeza, lo que en esta tristeza se pone al descubierto acerca de la "historia en total" es que la "unidad" del cuadro es precisamente el movimiento en 
sí mismo negador de todo hecho histórico determinado, esto es, finito. En otras palabras: que la historia, vista desde esta perspectiva como totalidad, es negación, y en consecuencia todo hecho histórico en rigor "no es".

Lo importante de la descripción, empero, es que ahora esta negatividad de la historia irrumpe en y como tristeza, es decir, que esta negatividad acontece realmente y es experimentada. Al contrario del contexto en donde esto aparece por primera vez, aquí no se trata ya sólo de una "categoría" a través de la cual lo histórico puede ser visto y asumido. Ciertamente esto también está implicado. Pero lo decisivo para el planteamiento filosófico es que lo que en esta tristeza hace su aparición, la "movilidad" de la historia como "negatividad", no pretende ser "negado" por Hegel, sino desplegado e interpretado en una dirección filosófica. La "negatividad" de la historia, esto es, el carácter "negativo" del proceso mismo en relación a lo determinado y finito en ese y por ese proceso, constituye en verdad un rasgo esencial de lo "histórico" desde el cual parte Hegel. La "negatividad" es, desde el punto del todo de acontecimientos y hechos históricos varios, algo esencial de la historia, en otras palabras, la historia no es ella misma ningún acontecimiento histórico, sino que "en total" una cierta "negación" de ellos. Lo notable de la descripción reside en que Hegel logra poner al descubierto como un estado de ánimo concreto lo que finalmente se abre en nosotros cuando tenemos ante nosotros el "espectáculo" de lo histórico. Justamente esta tristeza es ella misma la irrupción de esta negación.

Sin embargo, en esta "tristeza" ante lo histórico somos nosotros los que nos sentimos tristes. ¿Por qué? ¿Por qué justamente somos nosotros los que nos entristecemos? ¿Por qué el espectáculo de acontecimientos y generaciones completas de hombres que ya no son, puede en lo más mínimo llegar a afectarnos? Es cierto que Hegel en este pasaje no habla claramente de esto. Más bien lo que nos dice expresamente es que para quién está en esta experiencia, le está concedida incluso la posibilidad de "fortificarse" contra ella, esto es, puede quedar ajeno a ella mediante la constatación de lo "caduco" de todo "pasado", como, por ejemplo, cuando se afirma simplemente que "así ha sido" o que "no hay nada que podamos ya hacer". Justamente en la resignación con que se afirma esto, sólo se prepara la huida ante lo que ahí irrumpe. Siempre el observador finito de la historia podrá encubrirse a sí mismo el vínculo íntimo en el que él mismo se halla con respecto al todo de la historia, y de esta manera quedar librado a lo único que puede reconocer como real y efectivo en contra de la historia: los intereses y propósitos del presente. Librado así de 
la tristeza es como este observador puntual encuentra la perspectiva "segura" desde donde puede mirar como una "visión lejana" las ruinas que deben resultar siempre para él, en tanto inmerso en su presente, todo lo meramente "pasado", eso con lo que ya no hay nada qué hacer. Así mismo, refugiado en lo inmediato de sus intereses, incluso la "historia" misma puede ser tomada como una ocupación más, como algo con lo que se puede tener que ver sólo "desde lejos", esto es, con algo que en nada puede ya afectarme y que por tanto puede ser hurgado y trajinado en todas direcciones para darlo a conocer como "curiosidad". Ciertamente para el observador del caso es ésta una posibilidad concedida en tanto es este o aquel observador, pero no por eso deja de ser sólo una huida ante lo que desde el fondo mismo de la historia se alza: una innombrable tristeza sin posibilidad de consuelo o contrapeso: la historia como tal es negación.

Pero entonces, ¿¿de qué se huye en verdad aquí y por qué? Cuando Hegel pone de relieve esta "tristeza", cuando pensamos que en ella se trata de nosotros, y tan decisivamente que las más de las veces es necesario huir de ella para poder hacer "nuestras vidas en el presente", entonces esto indica que se trata de algo más que de la aparición fugaz de un cierto "estado de ánimo" ante el "espectáculo" de la historia al que tenemos acceso, por ejemplo, mediante la lectura de manuales o memorias. Pues sólo puede ser refugio el "presente" y sus intereses, esto es, sólo puede ser el lugar al que se llega en nuestro intento de huir, si justamente eso que concierne en total a todo acontecimiento histórico de manera negativa es lo que nos da la tristeza, nos toca y nos revela algo entristecedor de nosotros mismos. Lo que toca a todo acontecimiento histórico nos toca en el fondo a pesar de todo el empeño y vitalidad con que vivimos "sólo" en nuestro presente. Lo que por tanto nos entristece no es sólo que "por allá", en el pasado, todo acontecimiento es aniquilado por el propio movimiento de la historia, sino que toda nuestra actividad que se cree resuelta "sólo" en su presente y se satisface en esta ilusión, finalmente también ha de asistir a su ejecución en el "banco de verdugo" de la historia. En la experiencia con lo histórico nos exponemos por tanto a la "más honda tristeza", a esa que nos obliga a reconocer que de todo aquello en lo que ahora ponemos todo nuestro ser (inter-esse) finalmente no quedará nada, o en otras palabras, que todo aquello mediante lo cual pretendemos oponernos a esta tristeza y "seguir viviendo" no es sino finito y, en tanto tal, "caduco". Dándole la espalda a la historia sólo contribuimos a su inexorable movimiento. Por eso la experiencia de lo histórico pone en primer plano algo decisivo de ese observador: la tristeza y el dolor en el sentirse finito, que incluso en su intento de huir de esta tristeza 
sólo se entrega sin saberlo al inexorable movimiento que aniquila todo lo determinado, y con ello queda remitido de nuevo a su propia finitud.

\title{
3 Una justificación de la Filosofía de la Historia en relación a la EXPERIENCIA INTERPRETADA
}

Ahora bien, si esta interpretación de la descripción atina en lo esencial, tenemos que preguntar: ¿̇en qué medida está relacionado esto con la pregunta inicial, la pregunta que apunta a eso mismo de lo que la filosofía de la historia se encarga filosóficamente? Si nos fijamos tan sólo en el tono en que introduce la pregunta, éste contiene una cierta oposición a lo anterior:

\begin{abstract}
Aber auch indem wir die Geschichte als diese Schlachtbank betrachten, auf welchem das Glück der Völker, die Weisheit der Staaten und die Tugend der Individuen zum Opfer gebracht worden, so entsteht dem Gedanken notwendig auch die Frage, wem, welchem Endzwecke diese ungeheuersten Opfer gebracht worden sind ["Pero incluso en tanto consideremos la historia como el banco de verdugo en el cual han sido llevados a sacrificio la dicha de los pueblos, la sabiduría de los Estados y la virtud de los individuos, así le surge necesariamente al pensamiento también la pregunta: ¿para quién, para qué meta ha sido ofrecido este sacrificio inmenso?”] (HEGEL, 1955, p. 80).
\end{abstract}

No es que Hegel niegue de plano lo que se pone al descubierto de la historia en la tristeza, para luego pasar a la pregunta, sino que dice: "Pero incluso en tanto [...] así [...] necesariamente [...] también.” ¿Qué indica esto? Toda la frase comienza en verdad con una cierta "oposición" a lo anterior (aber), pero que no lo anula, sino que, por decirlo así, lo mira por otro lado. Ciertamente llegados al extremo de aquello que se pone al descubierto en la tristeza y el sentirnos tristes, teniendo que admitir la negatividad y la finitud que ahí se nos revela sin que quepa ya ninguna evasiva o huida ante ello, precisamente entonces, precisamente en ese "tanto" y justo en ello, asimismo también y a la vez le surge necesariamente al pensamiento la pregunta: ¿para qué? ¿Hacia dónde?

Sin embargo, ¿por qué justo en tanto que la tristeza pone al descubierto la negatividad de la historia necesariamente también le surge la pregunta "por la meta" al pensamiento? De esto el pasaje no da ninguna respuesta. Hegel aquí sólo dice que es así y que ciertamente es así "necesariamente". Pero ¿por qué? ¿De dónde viene la "necesidad" de que puestos en la experiencia de la 
negatividad de la historia a la vez también no surja la pregunta por su "meta"? Lo peculiar está en que, aun cuando estas preguntas puedan ellas plantearse legítimamente, ellas no se hacen cargo en rigor del modo en que el pensador expone el asunto. Pues de alguna forma estas preguntas que podemos hacernos parten del supuesto de que entre la experiencia descrita y la pregunta inicial hay una continuidad, es decir, que la necesidad del surgimiento de la pregunta está implicada en algo de la experiencia descrita hasta ahí. Y esto es precisamente lo que no pasa. Pues la pregunta para Hegel no surge desde la experiencia de la tristeza, como si fuese un paso más en la dirección de esta experiencia, sino que conjuntamente con ella, cuando renunciamos a toda huida'. Y ciertamente no le surge a ése que se haya en la tristeza en tanto anímicamente dispuesto, en tanto éste o este otro que ha descubierto en esta experiencia su propia finitud, sino en cuanto pensamiento. En cuanto individuo finito, que se tiene a sí mismo ante el espectáculo de la historia, alcanza lo extremo en la tristeza, pero en cuanto "pensar" no puede sino preguntar por aquello único que concierne a todo, universalmente, en cuanto hacia dónde, en cuanto "meta". En medio de esta pura tristeza sin posibilidad ya de evasiva, surge a la vez necesariamente la pregunta del pensamiento. Uno y otro a la vez, sin que haya derivación. De esta manera, la puesta al descubierto de experiencia es también y por su propia necesidad la apertura de lo otro, de lo no-finito, de lo in-finito y que como tal sólo puede ser cosa del pensar.

Entre uno y otro respecto no hay ninguna continuidad, sino que la frase en sí misma es el testimonio de un quiebre completo, justo desde el cual se pretende poner en marcha la Filosofía de la Historia en su necesidad y motivación más íntima. Y esto es tan así que hacia el final de este mismo pasaje es abandonada a su suerte esta "experiencia" cuando el mismo autor la declara como un "complacerse melancólicamente sobre las vanas e infecundas sublimidades de un puro resultado negativo" (HEGEL, 1955, p. 80). Esto significa que esta "experiencia" por sí misma no es esencial sino se retiene en ella esa pregunta que con ella surge pero que no tiene en ella su origen.

En este sentido el "origen" y "necesidad" misma de la pregunta pertenece para Hegel a un plano que esta misma experiencia no puede hacer ver, sino que refiere a un elemento "pensamental" del que la experiencia de un estado de ánimo no puede hacerse cargo. Dicho todavía con más precisión, la dirección no es continua desde esta experiencia hacia la pregunta que formula

\footnotetext{
${ }^{9}$ Se puede decir incluso: contra ella, tal como el mismo Hegel lo expone a reglón seguido (HEGEL,
} 1955, p. 80-81). 
el pensamiento, sino que lo que en verdad Hegel tiene a la vista es que recién con la irrupción de esta pregunta pensante sale a la luz el fondo desde el cual es posible la observación detenida de lo histórico, fondo que el observador en cuanto finito sólo es capaz de experimentar en la tristeza, como un puro "resultado negativo" en relación a su finitud. Sin embargo, en qué medida esto de "pensamiento" necesariamente interrumpe con las posibilidades concedidas a una "experiencia anímica" y abre una dimensión más amplia y fundamental del asunto, en qué medida, por tanto, es lo del pensamiento algo "anterior", no es algo que aquí podamos contestar cabalmente.

No obstante, de un supuesto como éste se nutre todo el intento de Hegel, si es que en verdad cabe aquí llamarlo "supuesto". Por el momento lo que a través de esta interpretación debemos asentar es que la negatividad del movimiento que es la historia no pretende ser negada por Hegel, sino más bien concebida filosóficamente según la dirección que marca la pregunta por una "meta" que surge al mismo tiempo que la experiencia en donde se revela esta negatividad en su aspecto inmediato. Sólo en tanto el origen y la necesidad de esta pregunta provienen del pensamiento, es por lo cual la filosofía y sólo ella es capaz de plantearla adecuadamente y responderla en verdad. Pero ¿qué significa esto? Lo que late ya en el sentido de la cuestión, tal como se orienta en ella Hegel, es que el propio movimiento negativo de la historia no es ni puede quedar en un "resultado meramente negativo", como en el estado anímico de la tristeza, no sólo porque el pensamiento filosófico sea lo único llamado a "concebirlo", sino porque en el fondo este movimiento mismo no tiene otro origen que "pensamiento", es el mismo en el modo del "pensar". La verdadera osadía con que Hegel acomete el asunto no reside sólo en "adaptar" una experiencia a preguntas y modos de preguntar que comprometen pensamiento, sino en ver que al fondo de esta experiencia, lo que se revela es ello mismo como "pensamiento" y "para el pensamiento" en la medida en que sólo aquí y recién aquí se disuelve la "finitud"10. Con la irrupción de la pregunta inicial sale a la luz lo primero, eso que, si sólo nos ceñimos a la experiencia de un observador finito, debe entristecernos y quedar como algo no verdaderamente concebido. La pregunta inicial filosóficamente planteada no anula por eso lo que esta experiencia pone al descubierto de la historia, sino que lo despliega en su verdadera "movilidad" y "negatividad"

\footnotetext{
${ }^{10}$ Llega a tal punto este condicionamiento, por decirlo así, lógico de la historia, que Hegel afirma en este texto: "El tiempo es negación, en lo sensible. El pensamiento es también negación; pero es la más íntima forma, la forma infinita en que todo ser se deshace y, en primer término, el ser finito, la forma definida" (HEGEL, 1955, p. 178).
} 
en la medida en que la filosofía es ella misma una concreción señalada del pensamiento. Es de esta manera como ya en medio de la experiencia con lo histórico en total aparece la Filosofía de la Historia justificada en su necesidad. Sólo ella es capaz de hacerse cargo, plantear y responder a eso que las más de la veces queda como algo meramente "observado" y repulsado como algo "lejano" y del "pasado", raras veces experimentado en total como algo de los que nos entristecemos y que desde su propio fondo nos "niega" en nuestra finitud, y muchas más raras asumido como pregunta que surge. De esta forma sólo para la Filosofía de la Historia, en tanto es la única capaz de concebir la historia desde su meta, la historia no es solo el paso (Gang) de lo que al punto se vuelve pasado y caduco (vergänglich), sino ante todo pro-greso (Fortgang).

\section{Consideraciones finales}

Resulta evidente que lo interpretado hasta aquí no es suficiente para una comprensión acabada de la Filosofía de la Historia de Hegel. Pero, como lo indicábamos al comienzo, no ha sido este el propósito. Es cierto que con la puesta al descubierto de la pregunta por la meta en su relación con la experiencia corriente de lo histórico, o incluso con el establecimiento de esta meta como "principio racional”, no se resuelve nada desde la perspectiva hegeliana. Bien al contrario, es tarea de la filosofía volverlo un auténtico principio, es decir, llevarlo a "su determinación precisa y a su desarrollo concreto" (HEGEL, 1955, p. 38). Es eso a lo que en rigor llama Hegel Filosofía de la Historia.

Sin embargo, de lo dicho podemos desatacar ciertos aspectos que permiten ver hasta qué punto se da en esta experiencia algo suficientemente radical acerca de lo histórico o hasta qué punto queda esto obstruido por la forma de disponerlo.

En primer lugar, llama la atención esa suerte de inquietud y desasosiego que sin mucho esfuerzo se puede percibir en dicha experiencia de lo histórico. En verdad, justo por aquí se puede ver a las claras la diferencia entre el modo de referencia con respecto a lo histórico en comparación con lo que sucede en la relación con lo natural. Y no se entiende bien esta inquietud si no poniendo de relieve a aquel que tiene y puede tener una relación expresa con lo histórico, es decir, a ese ente que está constituido como "espíritu". Por ello mismo, resulta a su vez llamativo, por no decir extraño, que de plano la "experiencia habitual" de lo histórico aparezca en la figura de una "observación” y "contemplación” del "cuadro de lo histórico". Sólo hasta cierto punto extrańo, pues es evidente que esta 
experiencia está dispuesta así, es decir, en primer lugar como una "contemplación de lo histórico", pues pesa para Hegel ese fenómeno al que en último término debe referir la Filosofía de la Historia. En otras palabras, la experiencia inmediata de lo histórico es presentada en el modo de una "contemplación", pues lo relevante es lo histórico en el sentido de "historia universal". Es de esto de lo que se busca "hacer filosofía". Por tanto, ahí donde lo histórico es identificado casi exclusivamente con la "historia universal" necesariamente la experiencia inmediata de lo histórico se ve reducida a una "contemplación”. De esta manera, poner en cuestión que la relación a lo histórico se lleve a cabo primeramente en el modo de un observar y contemplar debe ir aparejado con la puesta en cuestión de la identificación de lo histórico con la historia universal como fenómeno primario en que se ha de orientar la filosofía.

Sin embargo, esta forma de instalar la "experiencia habitual de lo histórico" puede parecer en principio extraño, aún si uno no sale del marco del propio Hegel. Pues, concediendo incluso lo dicho más arriba en términos generales, hay por lo menos dos momentos en la "Introducción a Filosofía de la Historia” en donde el filósofo alude a formas de relación con lo histórico en las que este carácter "observador" no es enteramente algo primario. Ya aparece en esa forma de "historia subjetiva"11, es decir, de conocimiento histórico que Hegel caracteriza como "historia originaria" (die urspüngliche Geschichte), pues precisamente lo distintivo de ella es que el vínculo que la une a lo que expone en la forma de un relato histórico emerge desde el vivir (Leben) y para el vivir. Es viviendo en medio de lo "histórico" por donde se gana el derecho a "conocerlo" y darlo a conocer. Y esto no sólo por haber sido testigo, sino en cuanto se tiene que actuar (handeln) en medio de ello y se ha tomado parte activa en esos acontecimientos. De ahí que Hegel ponga como ejemplos de "historiador originario" los casos de Heródoto, Tucídides y sobre todo César. Incluso el carácter discursivo del relato histórico es aquí peculiar, pues en efecto los discursos históricos son ellos mismos "las más esenciales y efectivas acciones" (HEGEL, 1955, p. 7), en el sentido en que la presentación que el historiador hace de lo acontecido esta destinada no en primer lugar a exhibir meramente los hechos, sino principalmente a disuadir en lo futuro de ciertas acciones o a incitar otras relevantes para la proyección de un cierto pueblo.

\footnotetext{
${ }^{11} \mathrm{El}$ tratamiento de esta forma aparece en el contexto mayor de exposición de las formas de abordar la historia (HEGEL, 1955, p. 1-23). Es de notar que esta exposición figura más bien en el proyecto de introducción del año 1822. Para ello se puede consultar los detalles de edición que da HOFFMEISTER (p. VII-XI, de la edición citada).
} 
Pero donde más se destaca la forma de experiencia histórica que no tiene su centro en la relación de contemplación es en el tratamiento de esa encarnación histórica que Hegel determina como "voluntad individual" y que denomina el "héroe". En palabras del pensador "Los grandes individuos en la historia universal son los que conciben lo general más alto y lo hacen su meta, son los que realizan esta meta, la cual es conforme al concepto superior del espíritu. Hay que nombrarlos héroes" (HEGEL, 1955, p. 97).

Es bien notorio aquí que el peso de la "experiencia de lo histórico" lo tiene la acción. Y esto incluso en la medida en que tiene por base aquello que Hegel entiende como el elemento en el cual se desarrolla la historia, es decir, lo que llama "voluntad". Es primariamente como "querer" que el individuo actúa históricamente. Sin embargo, resulta muy nítido precisamente en el tratamiento de la "voluntad individual" histórica la injerencia del planteamiento general que impone Hegel como legítimo para la filosofía de la historia. Pues, es aquí donde más perceptiblemente se ajusta lo de la categoría de "medio". Si consideramos lo limitado y finito de la conciencia de quien actúa históricamente en referencia a los alcances y al sentido completo de su acción ${ }^{12}$, o dicho de otro modo, si miramos el asunto destacando eso “animal" (pasión) que hay en la voluntad del "héroe" (HEGEL, 1955, p. 101) entonces esta voluntad individual no puede ser sino sólo el medio puesto en uso por "algo" que está más allá de ella y que da cuenta en total de la necesidad de esa acción. En este sentido no resulta extraño que precisamente en este contexto nos aparezca lo que el autor llama "el ardid de la razón" (die List der Vernunft): "La idea universal no se entrega a la contraposición y la lucha, no se expone al peligro; permanece intangible e ilesa, en el fondo, y envía a lo particular de la pasión a que reciba los golpes en la lucha... la razón hace que las pasiones obren por ella y que aquello que mediante la razón llega a la existencia, se pierda y sufra daño.” (HEGEL, 1955, p. 105).

Tal consideración de la voluntad individual como "medio" se explica, ciertamente, desde la posición fundamental del Filosofía de la Historia, a saber, la comprensión del desarrollo de la historia sobre el fondo de una meta que se realiza objetivamente como "Estado" a través de la serie de los diversos pueblos históricos. No es de extrańar, por ello, que tal como el propio Hegel

\footnotetext{
${ }^{12} \mathrm{El}$ ejemplo que pone Hegel para ilustrar esto es del que por venganza quema la casa de su enemigo, con lo cual inicia un movimiento cuyos "efectos" no están contenidos en la intención de la acción. El ejemplo aparece en: HEGEL (1955, p. 88-89).
} 
lo confiesa en varios pasajes ${ }^{13}$ la consideración filosófica de la historia incorpora elementos "lógicos", por lo pronto para estructurar el modelo de movilidad que impera también en la historia. Pero no sólo tiene la peculiar lógicafilosófica su incidencia en la articulación intrahistórica, sino que incluso la posición de esta misma en la dimensión "real del sistema", a saber, como el momento de consumación del "Espíritu objetivo" y por esto como tránsito al "absoluto" 14 , se puede ver al trasluz de la dialéctica propia de la "idea" en la absorción y superación del "querer" en la "idea absoluta"15. De esta forma, se puede afirmar no con mucho esfuerzo que precisamente la Filosofía de la Historia es en algún sentido la superación y absorción de la historia universal por la filosofía o, dicho de otro modo, la filosofía se ubica más bien en una situación "suprahistórica"16.

Ahora esa relación "contemplativa" con respecto a la historia tomada como historia universal se repite en un plano más fundamental (filosófico) que el que destacábamos con respecto a la "experiencia inmediata". Pero, esto no quiere decir que la filosofía no tenga cada vez una cierta situación histórica. Resulta bastante evidente que Hegel admite e incluye en el núcleo central de la filosofía como tal su carácter histórico ${ }^{17}$. Por eso, si se deja de lado el problema del carácter histórico de la propia Filosofía de la Historia y nos restringimos sólo a la historicidad de la filosofía en relación a la historia universal, entonces aparece a las claras que Hegel le asigna una situación "histórica" precisamente en cuanto momento se superación o, mejor dicho, de suspensión de las fuerzas que mueven el desarrollo de la historia universal en cuanto tal. De esto tenemos una serie de menciones en el propio texto de la "Introducción"18, e incluso desde aquí se puede leer ese célebre pasaje en que el pensador compara la situación histórica de la filosofía en relación a la historia universal como el "vuelo de la lechuza de Minerva" (HEGEL, 1979, p. 27-28).

Vemos, en definitiva, como la inmediata comprensión de la historia en el sentido de la historia universal nos lleva, primero, a fijar en un sentido

\footnotetext{
${ }^{13}$ Por lo menos en los siguientes pasajes: HEGEL (1955 p. 88-89, 155, 167).

${ }^{14}$ Consúltese para esto: HEGEL (1970, p. 366 ss).

${ }^{15}$ Consúltese para ello: HEGEL (1968, p. 554 ss).

${ }^{16}$ El término "suprahistórico" (Überhistorisch) es expresamente usado por Nietzsche para referirse a cierta historiografía de corte hegeliano. Consúltese NIETZSCHE (1930, p. 108-109, especialmente p. 166-168).

${ }^{17}$ Me refiero a la tan metada frase: "El estudio de la historia de la filosofía es el estudio de la filosofía misma" (HEGEL, 1972, p. 49).

${ }^{18}$ Por lo menos en los siguientes pasajes puede verse esto: HEGEL (1955, p. 71, 174).
} 
predominante la experiencia habitual de lo histórico en la forma de una experiencia de tipo "contemplativo", y seguidamente, a una paradójica situación histórica de la propia filosofía (suprahistórica). Finalmente sólo quisiera destacar que en el desarrollo del problema filosófico de la historia, por lo menos desde el punto de vista de cierta corriente filosófica cuyo punto de partida se puede fijar tal vez en Nietzsche que alcanza hasta el pensamiento de Heidegger, en la "experiencia de la historia" pierde relieve la "universalidad" de la historia para dar pie a la orientación según el vivir del individuo finito ${ }^{19}$. Con esta transformación del problema, que expresa o tácitamente va contra Hegel, también la situación histórica de la filosofía se vuelve una cuestión cada vez más acuciante y que justamente requiere para su asunción el abandono de la determinación de la filosofía como "saber absoluto" en el cual se disuelve la historia en cuanto se la concibe según la perspectiva de "metas" y "medios".

CEA, Francisco Abalo. An interpretation of a certain experience of the historical in Hegel's thought. Trans/Form/Açāo, Marília, v. 36, n. 2, p. 85-104, Maio/Ago., 2013.

\begin{abstract}
This article discusses the limits of the philosophy of history in Hegel's thought, centering on the interpretation of a certain experience of the historical that Hegel presents in his Introduction to the Philosophy of the History. There can be discovered in Hegel's exposition some typical elements of his philosophical position, but it is also possible to see up to what point his justification of the philosophy of the history is outlined in this text. Finally, we discuss some problematic aspects of the Hegelian position in light of our interpretation.
\end{abstract}

KEYWORDS: Philosophy of history, History, Experience, Spirit, Hegel.

\title{
Bibliografía
}

D'HONDT, Jacques. Hegel secret: recherchessur les sources cachées de la pensée de Hegel. Paris: Presses Universitaires de France, 1986.

FALAKE, G-H.H. Begriffne Geschichte. Das historische Substrat und die sistematische Andordnung der Bewußtseinsgehalten in Hegels Phänomenologie des Geistes. Berlin: Lukas, 1996. ${ }^{19}$ Ciertamente puede pensarse en esta dirección, por lo pronto, el trabajo de Nietzsche: "Vom Nutzen
und Nachteil der Historie für das Leben" (NIETZSCHE, 1930, p. 97-195). 
HEGEL, Georg.W.F. Die Vernunft in der Geschichte. En: Der Philosophischen Bibliotheke Band 171a. Hamburg: Felix Meiner, 1955.

Voltimedia, 2005.

Phänomenologie des Geistes. En: Hauptwerke der großen Denker. Bonn: . Wissenschaft der Logik, II Teil. En: Werke 6. Frankfurt am Main: Suhrkamp, 1968.

.Grundlinien der Philosophie des Rechts. En: Werke7. Frankfurt am Main: Suhrkamp, 1979.

. Enzyklopädie der philosphischen Wissenschaften, Teil II. En: Werke 8. Frankfurt am Main: Suhrkamp Verlag, 1970.

. Vorlesung über die Geschichte der Philophie, Teil I. En Werke 18. Frankfurt am Main: Suhrkamp, 1995.

HEIDEGGER, Martin. Phänomenologie des Religiösen Leben. En: Gesamtausgabe Band 60. Frankfurt am Main: Vittorio Klostermann, 1995.

Phänomenologie der Anschauung und Ausdrucks. En: Gesamtausgabe Band 60. Frankfurt am Main: Vittorio Klostermann, 1993.

MARCUSE, Herbert. Hegels Ontologie und die Grundlegung einer Theorie der Geschichtlichkeit. Frankfurt am Main: Vittorio Klostermann, 1932.

NIETZSCHE, Friedrich. Unzeitgemäße Betrachtungen. En: Band 71. Leipzig: Alfred Kröner, 1930.

Recebido em: 14.11.2012

Aceito em: 04.03.2013 
ABALO, F. 